ARTICLE

\title{
Quantized spin Hall conductance in a magnetically doped two dimensional topological insulator
}

\author{
Saquib Shamim (1) 1,2凶 , Wouter Beugeling (i) 1,2, Pragya Shekhar ${ }^{1,2}$, Kalle Bendias ${ }^{1,2}$, Lukas Lunczer ${ }^{1,2}$, \\ Johannes Kleinlein (10) ${ }^{1,2}$, Hartmut Buhmann ${ }^{1,2}$ \& Laurens W. Molenkamp (1) 1,2凶
}

Soon after the discovery of the quantum spin Hall effect, it has been predicted that a magnetic impurity in the presence of strong Coulomb interactions will destroy the quantum spin Hall effect. However, the fate of the quantum spin Hall effect in the presence of magnetic impurities has not yet been experimentally investigated. Here, we report the successful experimental demonstration of a quantized spin Hall resistance in $\mathrm{HgTe}$ quantum wells dilutely alloyed with magnetic $\mathrm{Mn}$ atoms. These quantum wells exhibit an inverted band structure that is very similar to that of the undoped material. Micron sized devices of $(\mathrm{Hg}, \mathrm{Mn}) \mathrm{Te}$ quantum well (in the topological phase) show a quantized spin Hall resistance of $h / 2 e^{2}$ at low temperatures and zero magnetic field. At finite temperatures, we observe signatures of the Kondo effect due to interaction between the helical edge channels and magnetic impurities. Our work lays the foundation for future investigations of magnetically doped quantum spin Hall materials towards the realization of chiral Majorana fermions.

\footnotetext{
${ }^{1}$ Experimentelle Physik III, Physikalisches Institut, Universität Würzburg, Am Hubland, Würzburg, Germany. ${ }^{2}$ Institute for Topological Insulators, Universität

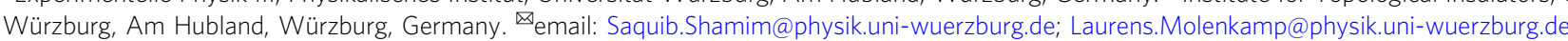


$\mathrm{T}$ he quantized spin Hall conductance is a hallmark of two dimensional (2D) topological insulators (TIs) where the conductance is solely due to a pair of counter propagating helical edge channels ${ }^{1-3}$. The stability of the quantum spin Hall (QSH) state in the presence of impurities has been the subject of numerous theoretical investigations ${ }^{4-18}$. For nonmagnetic impurities, which do not break time-reversal symmetry, the QSH states are robust against elastic single-particle backscattering events. Multi-particle scattering processes can, however, cause backscattering and hence lead to a suppression of the conductance away from the quantized value. Deviations from the quantized conductance of $2 e^{2} / h$ have been observed and attributed to various mechanisms including electron-electron interactions ${ }^{8}$, the presence of short range nonmagnetic impurities ${ }^{17}$ and tunneling to charge puddles ${ }^{9,12}$.

Magnetic impurities, on the other hand, can break time-reversal symmetry, and hence gap the QSH states. Maciejko et al. predicted that a magnetic impurity acting through the Kondo effect can lead to several interesting scenarios, depending on the strength of Coulomb interactions in the helical liquid ${ }^{6}$. For strong Coulomb interactions, two-particle backscattering processes ${ }^{6}$ lead to an insulating state at temperature $T=0$. For weak Coulomb interactions, the magnetic impurity causes spin-flip scattering when $T \gg$ $T_{\mathrm{K}}$, where $T_{\mathrm{K}}$ is the Kondo temperature, resulting in a logarithmic decrease of conductance with decreasing temperature. Upon decreasing the temperature, at $T \ll T_{\mathrm{K}}$ the conductance increases as a power law towards the quantized zero-temperature value $2 e^{2} / h^{6}$. Tanaka et al. show that the dc conductance is quantized to $2 e^{2} / h$ at all temperatures for isotropic Kondo interactions ${ }^{7}$. Anisotropic coupling of the spins of edge-state electrons to a macroscopic ensemble of magnetic impurities can lead to the spontaneous spin polarization of the impurities, resulting in the Anderson localization of edge states at $T=0^{11}$. Thus, there exist various theoretical predictions concerning the effect of magnetic impurities on the quantized conductance of a QSH insulator. Recent scanning tunneling microscopy experiments on one-dimensional topological edge states of bismuth, coupled to iron clusters, showed evidence of backscattering due to breakdown of time-reversal symmetry ${ }^{19}$. Such experiments, however, neither directly indicate the existence of a Kondo effect nor do they quantify the actual effect on the conductance quantization.

In spite of several theoretical predictions concerning the stability of the QSH effect, experimental investigations to test these predictions are rare, partly due to the limited number of available model systems, which demonstrate the quantized spin Hall conductance. A stable quantized plateau of $2 e^{2} / h$ has been successfully measured in transport experiments on nonmagnetic TIs based on $\mathrm{HgTe}$ quantum wells ${ }^{3}$, InAs/GaSb type-II quantum wells ${ }^{20}$ and monolayer $\mathrm{WTe}_{2}{ }^{21}$. Among the known quantum spin Hall systems, HgTe quantum wells provide an ideal platform to explore the rich phenomenology of the Kondo effect in magnetically doped TIs. This is because for dilute concentrations of Mn, the isoelectric substitution of $\mathrm{Mn}$ in $\mathrm{HgTe}$ causes neither additional carrier doping nor degradation in mobility. The weak Coulomb interactions in $\mathrm{HgTe}$ quantum wells ${ }^{6}$ suggest that $(\mathrm{Hg}, \mathrm{Mn}) \mathrm{Te}$ quantum wells are in the regime where Kondo physics due to the interaction of impurity spins with the helical edge channels can be investigated. A quantized spin Hall conductance in magnetically doped 2D TIs has, however, not yet been reported.

In this work, we report the observation of the quantum spin Hall effect in HgTe quantum wells with dilute concentration of $\mathrm{Mn}$ atoms. The longitudinal resistance is quantized to $h / 2 e^{2}$ at zero magnetic field. Temperature dependent measurements show that the quantized conductance is stable from 18 to $400 \mathrm{mK}$. At higher temperatures, the conductance decreases with increasing temperature, in agreement with ref. ${ }^{6}$, and is thus a signature of the Kondo effect due to interaction of helical edge channels and the spin of the magnetic impurity.

\section{Results and discussion}

Band structure calculated using the $\mathbf{k} \cdot \mathbf{p}$ method. In order to realize the QSH effect in a magnetically doped TI, we use a $(\mathrm{Hg}, \mathrm{Mn}) \mathrm{Te}$ quantum well that is $9 \mathrm{~nm}$ thick, with a $\mathrm{Mn}$ concentration of $1.2 \%$. The energy $E$ versus momentum $k$ dispersion relation for this quantum well is calculated using an 8 -orbital $\mathbf{k} \cdot \mathbf{p}$ method on a lattice for two scenarios: an infinite quantum well and a strip geometry ${ }^{22,23}$. In this model, we assume that the carriers couple to the average magnetization of the $\mathrm{Mn}$ ions (since their wave function covers a large number of $\mathrm{Mn}$ atoms), which responds paramagnetically to the external field at this concentration ${ }^{22,24}$. In absence of an external field, time-reversal symmetry is thus broken only at a microscopic level. Figure 1a shows the dispersion relation for an infinite $(\mathrm{Hg}, \mathrm{Mn}) \mathrm{Te}$ quantum well at zero-temperature and zero-magnetic field. We show the three subbands nearest to the gap, E1 with mixed $\Gamma_{6, \pm \frac{1}{2}}$ and $\Gamma_{8, \pm \frac{1}{2}}$ orbital character, and $\mathrm{H} 1$ and $\mathrm{H} 2$ with $\Gamma_{8, \pm \frac{3}{2}}$ orbital character, all of which are doubly degenerate. Due to the combination of the inverted gap of $\mathrm{HgTe}\left(\Gamma_{8, \pm \frac{3}{2}}\right.$ states at higher energy than the $\Gamma_{6, \pm \frac{1}{2}}$ states) and strain effects, the subbands are also inverted, with the H1 subband above the E1 subband. This band ordering, similar to that of undoped HgTe quantum wells in ref. ${ }^{3}$, implies the existence of a topological phase for a quantum well of the given thickness and concentration. The valence band (E1) shows maxima at finite momenta, which we refer to as the "camel back" feature. This feature is induced by a combination of the band inversion and hybridization between the subbands. The quantum well has an indirect band gap since the energy at the camel back is higher than the energy at $k=0$. From the band structure as function of $\left(k_{x}, k_{y}\right)$, shown in Fig. $1 \mathrm{~b}$, we find that the valence band maximum is reached at four points $k( \pm 1 / \sqrt{2}, \pm 1 / \sqrt{2})$, at the diagonal lines between the $k_{x}$ and $k_{y}$ axes. The dispersion of Fig. 1a is plotted along the line $\left(k_{x}, k_{y}\right)=k\left(\cos 45^{\circ}, \sin 45^{\circ}\right)=$ $k(1 / \sqrt{2}, 1 / \sqrt{2})$ so that the camel back shown in this figure represents the true maximum of the valence band.

Additionally, we have performed $\mathbf{k} \cdot \mathbf{p}$ calculations on a strip of width $500 \mathrm{~nm}$, having infinite length along the $x$-axis and spanning the range $y= \pm 250 \mathrm{~nm}$, in order to obtain the dispersions of the edge states. Figure 1c shows the band structure of the strip at zero magnetic field. The colors denote the location of the states along the width of the strip (see legend). One can clearly distinguish the edge states (red and blue) from the bulk states (gray). The Dirac point, where the edge states cross, is located just above the valence band at $k=0$.

Quantized spin Hall conductance in (Hg,Mn)Te quantum wells. The $(\mathrm{Hg}, \mathrm{Mn}) \mathrm{Te}$ quantum well used in this experiment has been grown by molecular beam epitaxy on lattice-matched $\mathrm{Cd}_{0.96} \mathrm{Zn}_{0.04} \mathrm{Te}$ substrate that provides minimal strain to the layers. The $(\mathrm{Hg}, \mathrm{Mn}) \mathrm{Te}$ quantum well is embedded between $\mathrm{Hg}_{0.3} \mathrm{Cd}_{0.7} \mathrm{Te}$ barriers with thicknesses of $53 \mathrm{~nm}$ (top barrier) and $158 \mathrm{~nm}$ (bottom barrier). A schematic of the layer stack is shown in Fig. 2a. The electron mobility for this quantum well is $\sim 2 \times$ $10^{5} \mathrm{~cm}^{2} \mathrm{~V}^{-1} \mathrm{~s}^{-1}$ at a carrier density of $n \sim 5 \times 10^{11} \mathrm{~cm}^{-2}$ as estimated from magneto-transport measurements in low perpendicular magnetic field.

Previous investigations on $\mathrm{HgTe}$ quantum wells have shown that the QSH effect is most easily observed in micron-sized Hall bars. We use a chemical wet-etching process to fabricate high mobility QSH microstructures in a six-terminal Hall bar 

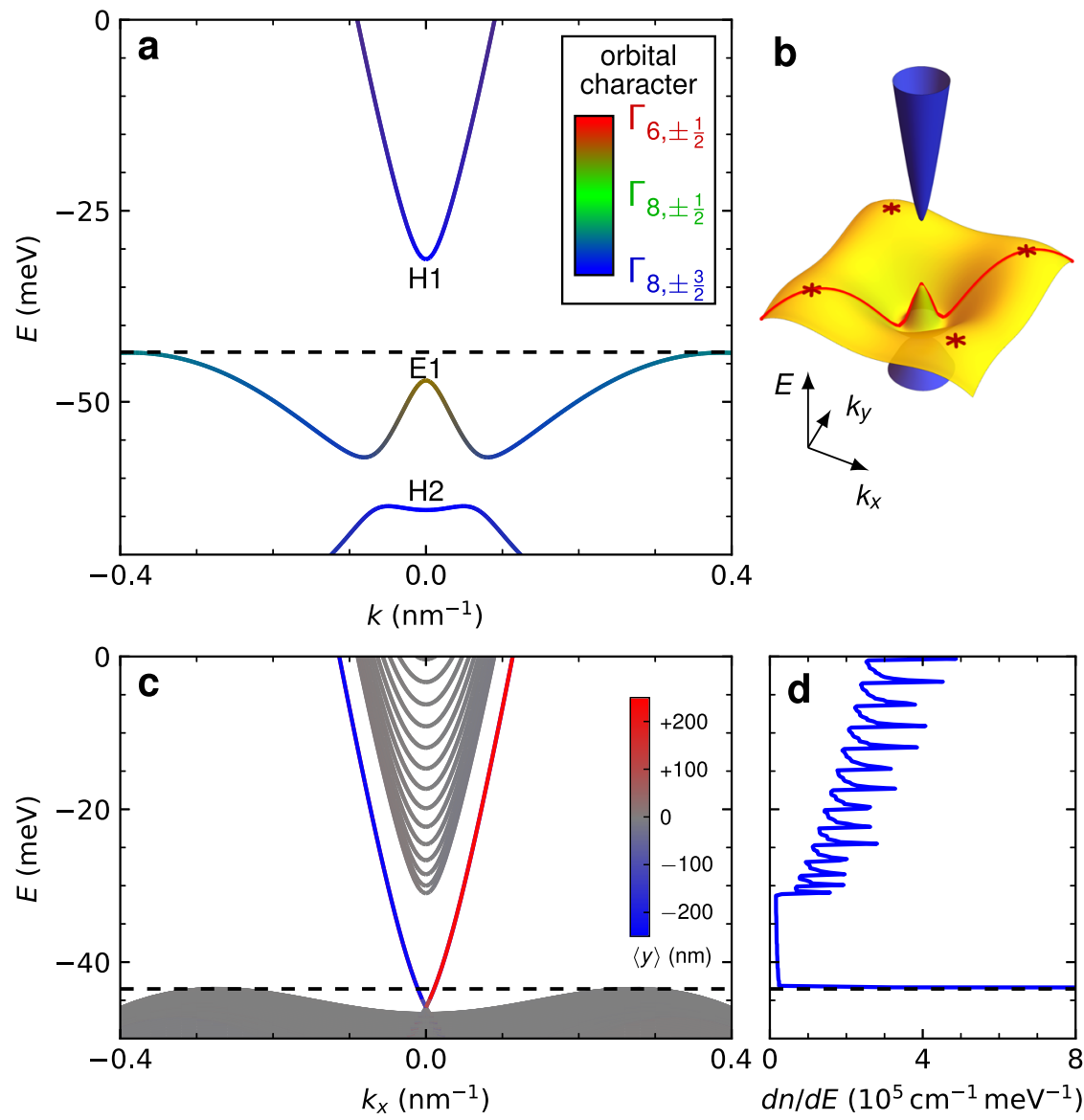

Fig. 1 Band structures calculated with the $\mathbf{k} \cdot \mathbf{p}$ method. a Dispersion for an infinite ( $\mathrm{Hg}, \mathrm{Mn}) \mathrm{Te}$ quantum well, $9 \mathrm{~nm}$ thick and with $1.2 \% \mathrm{Mn}$, along the axis $\left(k_{x}, k_{y}\right)=k\left(\cos 45^{\circ}, \sin 45^{\circ}\right)=k(1 / \sqrt{2}, 1 / \sqrt{2})$. The colors indicate the orbital character, see legend. $\mathbf{b}$ Three-dimensional rendering of the band structure. We highlight the axis of part $\mathbf{a}$, (red curve) and the camel back points (stars). c Dispersion in the strip geometry, $500 \mathrm{~nm}$ wide. The colors indicate the eigenstate location $\langle y\rangle$, see legend. $\mathbf{d}$ Density of carriers $d n / d E$ for the strip geometry. Just below the camel back ( $E \approx-46 \mathrm{meV}$, dashed lines), the density reaches values $>400 \times 10^{5} \mathrm{~cm}^{-1} \mathrm{meV}^{-1}$, out of scale in this plot.

a

\section{$\mathrm{Cd}_{0.7} \mathrm{Hg}_{0.3} \mathrm{Te}(53 \mathrm{~nm})$ \\ $(\mathrm{Hg}, \mathrm{Mn}) \mathrm{Te} 9 \mathrm{~nm}, 1.2 \%$ \\ $\mathrm{Cd}_{0.7} \mathrm{Hg}_{0.3} \mathrm{Te}(158 \mathrm{~nm})$ \\ CdTe Buffer $(50 \mathrm{~nm})$ \\ $\mathrm{Cd}_{0.96} \mathrm{Zn}_{0.04} \mathrm{Te}$ Substrate}

b

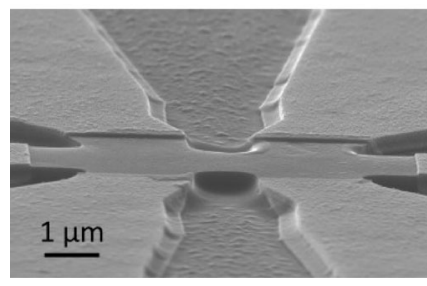

C

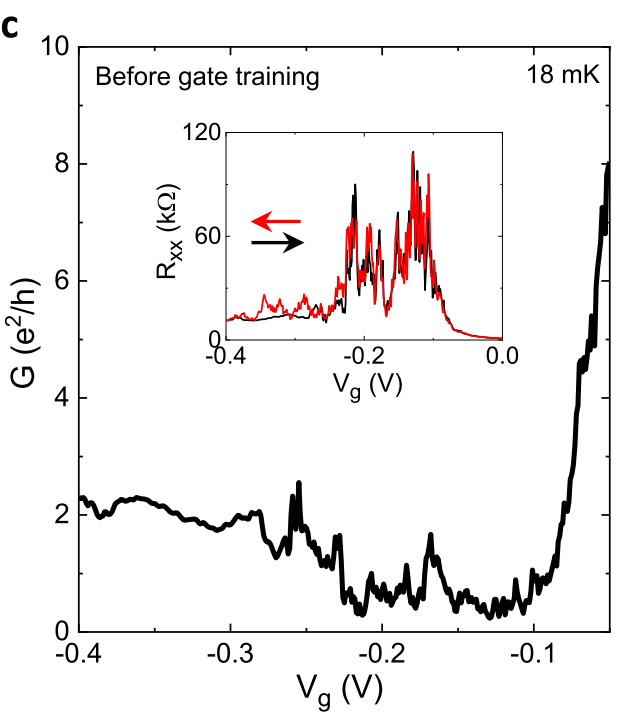

d

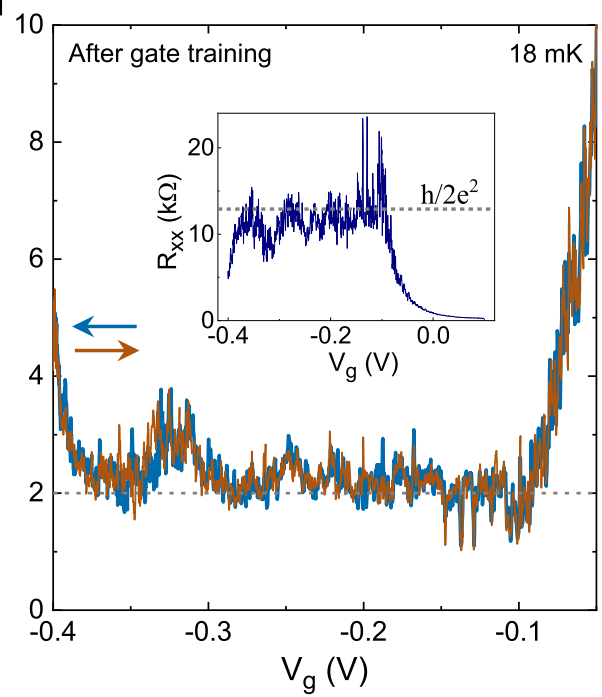

Fig. 2 Quantized spin Hall conductance in a ( $\mathbf{H g}, \mathbf{M n})$ Te quantum well. a Schematic of the layer stack showing the (Hg,Mn)Te quantum well sandwiched between two $\mathrm{Cd}_{0.7} \mathrm{Hg}_{0.3} \mathrm{Te}$ barriers on a $\mathrm{Cd}_{0.96} \mathrm{Zn}_{0.04} \mathrm{Te}$ substrate. b Scanning electron micrograph of a typical Hall bar used for measurements. c The conductance $G$ as a function of gate voltage $V_{g}$ for a $9 \mathrm{~nm}$ thick $(\mathrm{Hg}, \mathrm{Mn}) \mathrm{Te}$ quantum well with $1.2 \% \mathrm{Mn}$ at $18 \mathrm{mK}$. The inset shows the longitudinal resistance $R_{x x}$ as a function of $V_{g}$. d $G$ as a function of $V_{g}$ at $18 \mathrm{mK}$ after gate training. The inset shows $R_{x x}$ as a function of $V_{g}$. The dotted line indicates the expected conductance of $2 e^{2} / h$ in the quantum spin Hall regime. The arrows in $\mathbf{c}$ and $\mathbf{d}$ show the direction of gate voltage sweeps. 
geometry of dimensions $1.9 \times 1.7 \mu \mathrm{m}^{2}$, with the same crystal quality as the original MBE grown material ${ }^{25}$. A $14 \mathrm{~nm}$ thick $\mathrm{HfO}_{2}$ layer grown by atomic layer deposition is used as a dielectric insulator. The carrier density can be controlled by applying a voltage to a $5 / 200 \mathrm{~nm} \mathrm{Ti} / \mathrm{Au}$ gate layer deposited on the $\mathrm{HfO}_{2}$ insulator. The ohmic contacts are formed by depositing $50 \mathrm{~nm}$ of $\mathrm{AuGe}$ and $50 \mathrm{~nm}$ of Au by e-beam evaporation. More details of the device fabrication process can be found in ref. ${ }^{25}$. A scanning electron micrograph of a typical micro Hall bar (without the top gate) is shown in Fig. $2 b$.

All electrical transport measurements have been performed in a dilution refrigerator at a base temperature of $18 \mathrm{mK}$. The resistance of the device has been measured in a four terminal configuration using low frequency $(\approx 13 \mathrm{~Hz})$ lock-in techniques. The device can be tuned from $n$-type conduction for $V_{g} \geq 0 \mathrm{~V}$ to $p$-type conduction for $V_{g} \leq-0.4 \mathrm{~V}$ (Fig. 2c). The bulk band gap regime is identified by an increased resistance (or decreased conductance in Fig. 2c) for $V_{g}=-0.1$ to $-0.2 \mathrm{~V}$ (inset of Fig. 2c). We note that in the bulk gap regime $R_{x x}$ is much larger than the expected quantized resistance of $h / 2 e^{2}$. We have recently shown that such a deviation from the quantized value results from charge puddle induced potential fluctuations ${ }^{26}$. In the present sample, the resistance (when the chemical potential is in the bulk band gap) shows large fluctuations ( $\sim$ tens of $\mathrm{k} \Omega$ ) for the virgin gate sweep, which is a characteristic of our Mn-doped HgTe devices. For undoped HgTe structures, the fluctuations in the quantized spin Hall resistance are much smaller $\sim 1 \mathrm{k} \Omega^{3,25}$. The measured fluctuations in $R_{x x}$ are observed only in microscopic devices as they are due to scattering from a small number of charge puddles. As has been shown in ref. ${ }^{26}$, the average distance between puddles is $\sim$ few $\mu \mathrm{m}$, which implies that a micron-sized device is affected by only a few charge puddles. A comprehensive analysis of the conductance fluctuations and their dependence on temperature will be discussed in a future publication.

Reference $^{26}$ also demonstrates that in our HgTe samples it is possible to smooth out the potential landscape and thus approach the expected QSH resistance by repeated gate sweeps ("gate training"). We follow a similar procedure and sweep the gate voltage multiple times $(\sim 300)$ between 0.1 and $-0.4 \mathrm{~V}$, the range for which we do not observe any gate hysteresis. The gate voltage characteristics of the device at $18 \mathrm{mK}$ after gate training show an excellent quantization of the conductance $G$ to $2 e^{2} / h$ for a range of $V_{g}$ from $-0.1 \mathrm{~V}$ to $-0.3 \mathrm{~V}$ (Fig. 2d). The inset of Fig. 2d shows the quantization in resistance as a function of $V_{g}$ at $18 \mathrm{mK}$. Apart from the conductance quantization, another noticeable effect of gate training is that the fluctuations in resistance are now of the order $\sim \mathrm{k} \Omega$ (inset of Fig. $2 \mathrm{~d}$ ), an order of magnitude smaller as compared to the device before gate training. The observation of a quantized conductance and reduced fluctuations after gate training is clear evidence that we have achieved a smoother potential landscape. The quantized spin Hall conductance in a magnetically doped 2D TI at zero magnetic field clearly indicates that the edge channels are robust also in the presence of magnetic impurities. This is the key observation of the paper. The quantized conductance due to quantum spin Hall effect has been reproduced in this device after a thermal cycle and in other devices fabricated from the same wafer. Devices with different Mn concentrations $(<2.5 \%)$ have also shown the conductance quantization due to the quantum spin Hall effect.

Temperature dependence of the quantized spin Hall conductance: signature of the Kondo effect. To probe the temperature limit up to which the QSH effect persists in our magnetically doped TIs, we have performed transport measurements at various temperatures. The $G-V_{g}$ traces at temperatures ranging from $18 \mathrm{mK}$ to $4.2 \mathrm{~K}$ (Fig. 3a) show several distinct features. The fluctuations in conductance are reproducible at different temperatures (Fig. 3b) and hence are not an artefact of measurement noise. The fluctuations in conductance are largest at lowest temperature and decrease as the temperature increases. The average conductance in the QSH regime, $G_{\text {gap }}^{\text {av }}$, is obtained by averaging over the conductance values in the plateau region of the $G-V_{g}$ curve (region between the vertical dashed lines in the inset of Fig. $3 c$, note that the length of the plateau region decreases at higher temperatures). The error in determining $G_{\text {gap }}^{\text {av }}$ at a particular temperature has been estimated by calculating the standard deviation of the $G_{\text {gap }}^{\text {av }}$ values obtained by choosing multiple $(>5)$
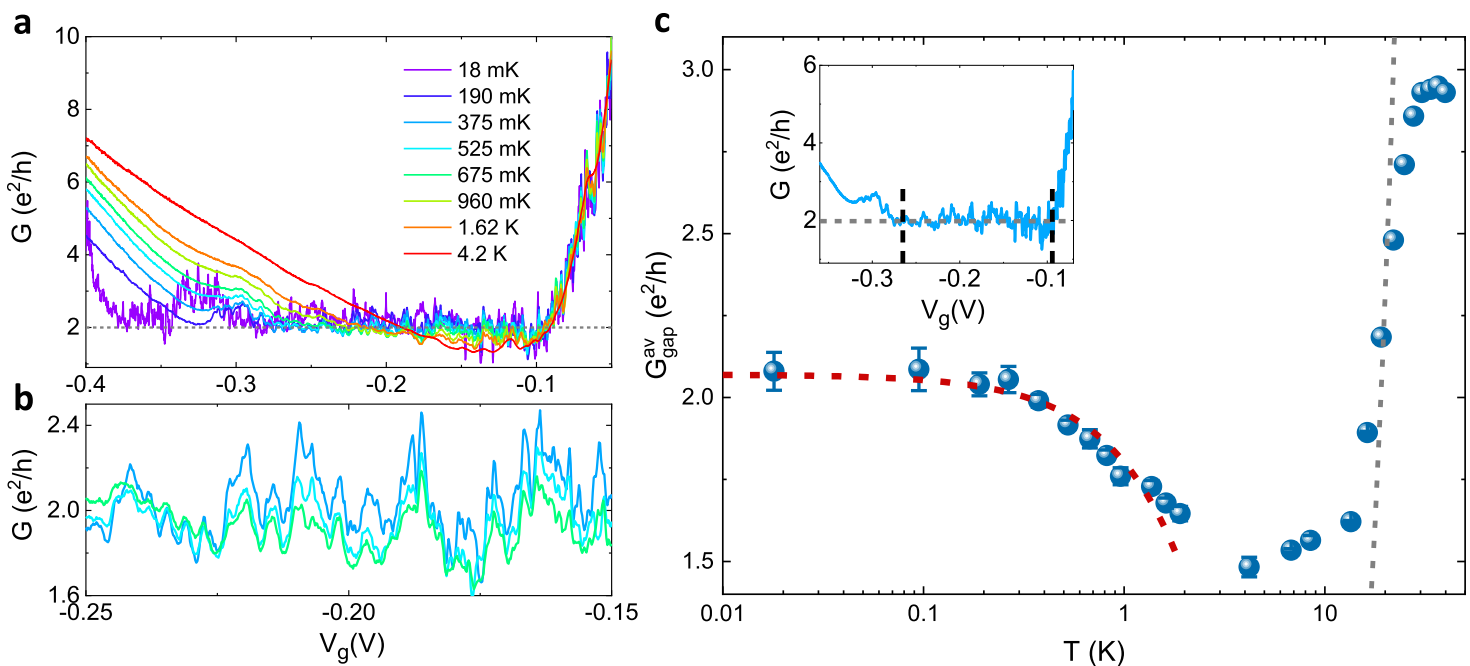

Fig. 3 Effect of temperature on the quantized conductance. a The conductance $G$ as a function of gate voltage $V_{g}$ at different temperatures from $18 \mathrm{mK}$ to $4.2 \mathrm{~K}$. The dotted line indicates the expected conductance of $2 e^{2} / \mathrm{h}$ in the quantum spin Hall regime. $\mathbf{b}$ Magnified view of $G$ as a function $V_{g}$ for different temperatures to show the reproducibility in conductance fluctuations. c The average conductance in the quantum spin Hall regime $G_{\text {gap }}^{\text {av }}$ as a function of temperature $T$. The blue points are the experimental data and the red dashed line shows the power-law Kondo fit (see main text). The gray dashed line shows the expected thermally activated conductance $\left[\propto \exp \left(-E_{g} / 2 k_{B} T\right)\right]$ for a band gap of $E_{g}=10 \mathrm{meV}$ (from the band structure calculations shown in Fig. 1). The inset shows a typical $G-V_{g}$ dependence from which $G_{\text {gap }}^{\text {av }}$ (shown by dashed horizontal line) is calculated by averaging over the conductance values in the plateau region between the dashed vertical lines. 
realistic plateau regions of the $G-V_{g}$ curve. The average conductance in the QSH regime is constant up to $400 \mathrm{mK}$ and subsequently decreases with increasing temperature (blue points in Fig. 3c). This observation is qualitatively in agreement with ref. ${ }^{6}$, where it is predicted that for weak Coulomb interactions, the magnetic impurity spin is fully screened by the edge electrons at $T=0$ and the conductance is quantized to $2 e^{2} / h$. An increase in temperature leads to a decrease in conductance because the impurity spin is not fully screened at higher temperatures and causes inelastic scattering. The observation of quantized conductance at low temperatures and subsequent decrease in conductance at higher temperature $(>400 \mathrm{mK})$ is a manifestation of the Kondo effect due to interaction of helical edge channels with the magnetic impurities. In contrast, for HgTe quantum wells without $\mathrm{Mn}$ doping, we have observed quantized conductance up to $15 \mathrm{~K}^{25}$. While a spin glass phase due to antiferromagnetic ordering of $\mathrm{Mn}$ has been observed for II-VI compounds doped with high concentration of $\mathrm{Mn}(7-20 \%)$ at millikelvin temperatures ${ }^{27}$, which can cause scattering and fluctuations in conductance, we observe a quantized conductance from $18-400 \mathrm{mK}$ and hence can rule out scattering due to antiferromagnetic ordering at low temperatures.

From refs. ${ }^{5,6}$, for weak Coulomb interactions, the decrease of conductance with increasing temperature follows a power law $G=G_{0}-\alpha\left(T / T_{\mathrm{K}}\right)^{2(4 K-1)}$, where $G_{0}$ is the conductance due to quantized spin Hall edge channels, $\alpha$ is a proportionality constant and the interaction parameter $K$ quantifies the strength of Coulomb interactions in the helical edge channels. For HgTe quantum wells, the Coulomb interactions are expected to be weak ${ }^{28,29}$. Hence we fit the low temperature part of the $G_{\text {gap }}^{\text {av }}(T)$ dependence with the expression $G=G_{0}-\alpha\left(T / T_{\mathrm{K}}\right)^{2(4 K-1)}$ (red dashed curve in Fig. $3 \mathrm{c}$ is the fit) and obtain a reasonable value of $K \sim 0.4$. The proportionality constant $\alpha$ was set to $e^{2} / h, G_{0}=$ $2.07 e^{2} / h$ and $T_{\mathrm{K}} \sim 3 \mathrm{~K}$. Since this is a multi-parameter fit for a rather limited range of temperature, we cannot conclusively rule out other mechanisms of temperature dependent corrections to the QSH conductance. However, samples without Mn doping do not show the pronounced decrease in conductance with increasing temperature in the range of $100 \mathrm{mK}$ to a few $\mathrm{K}$ observed here. In Mn-free samples, the quantized conductance due to the quantum spin Hall effect is temperature independent up to $T \sim 15 \mathrm{~K}^{25}$. Hence, the initial decrease in conductance in the sub-Kelvin range in Fig. $3 \mathrm{c}$ is a strong indication of the Kondo origin of this observation. An independent estimate of $K$ can be obtained from the formula ${ }^{28}$,

$$
K=\left[1+\frac{2}{\pi} \frac{e^{2}}{\epsilon_{0} \epsilon_{r} \hbar v_{F}} \ln \left(\frac{7.1 d}{\xi+0.8 w}\right)\right]^{-1 / 2}
$$

where $\epsilon_{0}$ is the permittivity of free space, $\epsilon_{r}$ is the dielectric constant, $v_{F}$ is the Fermi velocity, $d$ is the distance between the edge channel and the gate, $w$ is the thickness of the quantum well, $\xi=2 \hbar v_{F} / E_{\text {gap }}$ and $E_{\text {gap }}$ is the band gap of the system. Using $\epsilon_{r}=20$ for $\mathrm{HgTe}, v_{F}=5.5 \times 10^{5} \mathrm{~m} \mathrm{~s}^{-1}, d=70 \mathrm{~nm}, w=9 \mathrm{~nm}$, and $E_{g a p}=$ $10 \mathrm{meV}$, we get $K \sim 0.5$, which is in good agreement with the value obtained by fitting the experimental data in Fig. $3 \mathrm{c}$. For $T>T_{\mathrm{K}}$, the conductance increases slowly with increasing temperature (up to $\sim 15 \mathrm{~K})$ as expected for spin-flip scattering off a magnetic impurity ${ }^{6}$. Above $15 \mathrm{~K}$, the conductance increases sharply with increasing temperature since the thermal excitations across the bulk band gap of $10 \mathrm{meV}$ (from $\mathbf{k} \cdot \mathbf{p}$ band structure calculations shown in Fig. 1) strongly contribute to the measured conductance. To illustrate this, we show in Fig. 3c (gray dashed line), the expected thermally activated conductance $\left[\propto \exp \left(-E_{g} / 2 k_{B} T\right)\right]$ for a band gap of $E_{g}=10 \mathrm{meV}$, which agrees with the experimentally observed behavior. Hence, for higher temperatures, the conductance increases above the expected quantized value. The saturation in conductance for $T>30 \mathrm{~K}$ is due to enhanced phonon scattering at high temperatures ${ }^{30}$.

In addition, we observe that the length of the quantized plateau (in $V_{g}$ ) in Fig. 3a decreases strongly with increasing temperature. For the $G-V_{g}$ curves shown in Fig. 3a, there is no "isosbestic point" (the curves for different temperatures do not cross at the same point). This suggests that the temperature dependence of conductance for large negative $V_{g}$ is determined by processes, which are different from those described in the previous paragraph for the temperature dependence of $G_{\text {gap }}^{\text {av }}$. To understand the temperature dependence of the length of the quantized plateau and the conductance for large negative $V_{g}$, we have calculated the density of states from the dispersion shown in Fig. 1c. There is an extremely large density of states in the valence band, arising from the camel back, which is $~ 50-100$ times larger than that in the conduction band (Fig. 1d). This large density of states near the camel back causes 'pinning' of the chemical potential, i.e., the position of the chemical potential is almost unchanged for a large range of gate voltages. Even though the camel back (bulk) states are occupied, they do not contribute to the conductance at lowest temperatures $(18 \mathrm{mK})$ as the carriers are localized by disorder ${ }^{23}$. Hence the conductance is quantized to the QSH value of $2 e^{2} / h$ for a large range of gate voltage at $18 \mathrm{mK}$ (Fig. 2c). We have shown in ref. ${ }^{23}$, that for higher temperatures the carriers in the camel back are delocalized. Hence, the measured longitudinal conductivity rises above $2 e^{2} / h$ from contributions of bulk states at the top of the camel back, resulting in a strong increase of conductance with increasing temperature for $V_{g}<-0.3 \mathrm{~V}$.

To summarize, we have performed transport measurements in a magnetically doped TI realized by incorporating $\mathrm{Mn}$ atoms in a HgTe quantum well. The conductance of the micron-sized Hall bar is quantized to $2 e^{2} / h$ up to $400 \mathrm{mK}$ and subsequently decreases with increasing temperatures, confirming the prediction of interaction of helical edge channels with a Kondo impurity ${ }^{6}$. Our observation of quantized conductance in $(\mathrm{Hg}, \mathrm{Mn}) \mathrm{Te}$ quantum wells should lead to further investigations of the Kondo effect in magnetically doped 2D TIs, particularly in the presence of tunable Rashba interaction which can control the Kondo temperature ${ }^{31}$, thus making the Kondo crossover (the regime for $T \gg T_{\mathrm{K}}$ where conductance increases with increasing temperature) accessible in the transport experiments. Transport investigations in devices patterned in Corbino geometry can give further insights of the edge-state transport in magnetically doped quantum spin Hall materials. The realization of quantized spin Hall conductance in a magnetically doped TI lays the groundwork for further experiments on Majorana fermions by interfacing these structures with superconductors.

\section{Data availability}

All data necessary to support the conclusions of the paper are available in the manuscript. Source data are provided with this paper.

\section{Code availability}

The code used for the band structure analysis is available from the corresponding authors upon reasonable request.

Received: 11 September 2020; Accepted: 21 April 2021; Published online: 27 May 2021

\section{References}

1. Kane, C. L. \& Mele, E. J. $Z_{2}$ topological order and the quantum spin Hall effect. Phys. Rev. Lett. 95, 146802 (2005). 
2. Bernevig, B. A., Hughes, T. L. \& Zhang, S.-C. Quantum spin Hall effect and topological phase transition in HgTe quantum wells. Science 314, 1757-1761 (2006).

3. König, M. et al. Quantum spin Hall insulator state in $\mathrm{HgTe}$ quantum wells. Science 318, 766-770 (2007)

4. Wu, C., Bernevig, B. A. \& Zhang, S.-C. Helical liquid and the edge of quantum spin Hall systems. Phys. Rev. Lett. 96, 106401 (2006).

5. Xu, C. \& Moore, J. E. Stability of the quantum spin Hall effect: Effects of interactions, disorder, and $Z_{2}$ topology. Phys. Rev. B 73, 045322 (2006).

6. Maciejko, J. et al. Kondo effect in the helical edge liquid of the quantum spin Hall state. Phys. Rev. Lett. 102, 256803 (2009).

7. Tanaka, Y., Furusaki, A. \& Matveev, K. A. Conductance of a helical edge liquid coupled to a magnetic impurity. Phys. Rev. Lett. 106, 236402 (2011).

8. Schmidt, T. L., Rachel, S., von Oppen, F. \& Glazman, L. I. Inelastic electron backscattering in a generic helical edge channel. Phys. Rev. Lett. 108, 156402 (2012).

9. Väyrynen, J. I., Goldstein, M. \& Glazman, L. I. Helical edge resistance introduced by charge puddles. Phys. Rev. Lett. 110, 216402 (2013).

10. Cheianov, V. \& Glazman, L. I. Mesoscopic fluctuations of conductance of a helical edge contaminated by magnetic impurities. Phys. Rev. Lett. 110, 206803 (2013).

11. Altshuler, B. L., Aleiner, I. L. \& Yudson, V. I. Localization at the edge of a 2D topological insulator by Kondo impurities with random anisotropies. Phys. Rev. Lett. 111, 086401 (2013).

12. Väyrynen, J. I., Goldstein, M., Gefen, Y. \& Glazman, L. I. Resistance of helical edges formed in a semiconductor heterostructure. Phys. Rev. B 90, 115309 (2014).

13. Kimme, L., Rosenow, B. \& Brataas, A. Backscattering in helical edge states from a magnetic impurity and Rashba disorder. Phys. Rev. B 93, 081301 (2016).

14. Väyrynen, J. I. \& Glazman, L. I. Current noise from a magnetic moment in a helical edge. Phys. Rev. Lett. 118, 106802 (2017).

15. Zheng, J.-H. \& Cazalilla, M. A. Nontrivial interplay of strong disorder and interactions in quantum spin-Hall insulators doped with dilute magnetic impurities. Phys. Rev. B 97, 235402 (2018).

16. Väyrynen, J. I., Pikulin, D. I. \& Alicea, J. Noise-induced backscattering in a quantum spin Hall edge. Phys. Rev. Lett. 121, 106601 (2018).

17. Novelli, P., Taddei, F., Geim, A. K. \& Polini, M. Failure of conductance quantization in two-dimensional topological insulators due to nonmagnetic impurities. Phys. Rev. Lett. 122, 016601 (2019).

18. Kurilovich, V. D., Kurilovich, P. D., Burmistrov, I. S. \& Goldstein, M. Helical edge transport in the presence of a magnetic impurity: The role of local anisotropy. Phys. Rev. B 99, 085407 (2019).

19. Jäck, B., Xie, Y., Bernevig, B. A. \& Yazdani, A. Observation of backscattering induced by magnetism in a topological edge state. Proc. Natl Acad. Sci. USA 117, 16214-16218 (2020)

20. Knez, I., Du, R.-R. \& Sullivan, G. Evidence for helical edge modes in inverted InAs/GaSb quantum wells. Phys. Rev. Lett. 107, 136603 (2011).

21. Wu, S. et al. Observation of the quantum spin Hall effect up to 100 kelvin in a monolayer crystal. Science 359, 76-79 (2018).

22. Novik, E. G. et al. Band structure of semimagnetic $\mathrm{Hg}_{1-y} \mathrm{Mn}_{y} \mathrm{Te}$ quantum wells. Phys. Rev. B 72, 035321 (2005).

23. Shamim, S. et al. Emergent quantum Hall effects below $50 \mathrm{mt}$ in a twodimensional topological insulator. Sci. Adv. 6, eaba4625 (2020).

24. Hui, P. M., Ehrenreich, H. \& Hass, K. C. Effects of $d$ bands on semiconductor sp hamiltonians. Phys. Rev. B 40, 12346-12352 (1989).

25. Bendias, K. et al. High mobility HgTe microstructures for quantum spin Hall studies. Nano Lett. 18, 4831-4836 (2018).

26. Lunczer, L. et al. Approaching quantization in macroscopic quantum spin Hall devices through gate training. Phys. Rev. Lett. 123, 047701 (2019).

27. Jaroszyński, J., Wróbel, J., Karczewski, G., Wojtowicz, T. \& Dietl, T. Magnetoconductance noise and irreversibilities in submicron wires of spinglass $n^{+}-\mathrm{Cd}_{1-x} \mathrm{Mn}_{x}$ Te. Phys. Rev. Lett. 80, 5635-5638 (1998).
28. Teo, J. C. Y. \& Kane, C. L. Critical behavior of a point contact in a quantum spin Hall insulator. Phys. Rev. B 79, 235321 (2009).

29. Strunz, J. et al. Interacting topological edge channels. Nat. Phys. 16, 83-88 (2020).

30. Leubner, P., Lunczer, L., Brüne, C., Buhmann, H. \& Molenkamp, L. W. Strain engineering of the band gap of $\mathrm{HgTe}$ quantum wells using superlattice virtual substrates. Phys. Rev. Lett. 117, 086403 (2016)

31. Eriksson, E., Ström, A., Sharma, G. \& Johannesson, H. Electrical control of the Kondo effect in a helical edge liquid. Phys. Rev. B 86, 161103 (2012).

\section{Acknowledgements}

We thank J. Väyrynen and C. Gould for useful discussions. We acknowledge financial support from the Deutsche Forschungsgemeinschaft (DFG, German Research Foundation) in the Leibniz Program and in the projects SFB 1170 (Project ID 258499086) and SPP 1666 (Project ID 220179758), from the EU ERC Advanced Grant “4-TOPS” (Project 741734), from the Würzburg-Dresden Center of Excellence 'Complexity \& Topology in Quantum Matter' (CT.QMAT), and from the Free State of Bavaria (Elitenetzwerk Bayern IDK 'Topologische Isolatoren' and the Institute for Topological Insulators).

\section{Author contributions}

S.S., H.B., and L.W.M. planned the experiments. S.S. conducted the measurements with help from P.S. and analyzed the data. W.B. performed the band structure calculations. L.L. grew the material. K.B. and P.S. fabricated the device. J.K. oversaw the device fabrication. All authors contributed to interpretation of the results. L.W.M. and H.B. supervised the project. S.S. and W.B. wrote the paper with input from all authors.

\section{Funding}

Open Access funding enabled and organized by Projekt DEAL.

\section{Competing interests}

The authors declare no competing interests.

\section{Additional information}

Supplementary information The online version contains supplementary material available at https://doi.org/10.1038/s41467-021-23262-1.

Correspondence and requests for materials should be addressed to S.S. or L.W.M.

Peer review information Nature Communications thanks the anonymous reviewer(s) for their contribution to the peer review of this work.

Reprints and permission information is available at http://www.nature.com/reprints

Publisher's note Springer Nature remains neutral with regard to jurisdictional claims in published maps and institutional affiliations.

\begin{abstract}
cc) (i) Open Access This article is licensed under a Creative Commons By Attribution 4.0 International License, which permits use, sharing adaptation, distribution and reproduction in any medium or format, as long as you give appropriate credit to the original author(s) and the source, provide a link to the Creative Commons license, and indicate if changes were made. The images or other third party material in this article are included in the article's Creative Commons license, unless indicated otherwise in a credit line to the material. If material is not included in the article's Creative Commons license and your intended use is not permitted by statutory regulation or exceeds the permitted use, you will need to obtain permission directly from the copyright holder. To view a copy of this license, visit http://creativecommons.org/ licenses/by/4.0/.
\end{abstract}

(c) The Author(s) 2021 